\title{
Physics of Breakup in Liquid Column and Sheet
}

\author{
M. Ali*a, M. Q. Islam ${ }^{a}$ and M. Khadem ${ }^{b}$ \\ ${ }^{a}$ Department of Mechanical Engineering, Bangladesh University of Engineering and Technology, Dhaka - 1000, \\ Bangladesh \\ ${ }^{b}$ Department of Mechanical and Industrial Engineering, College of Engineering, Sultan Qaboos University, P.O. Box 33, \\ Postal Code 123, Al Khoud, Muscat, Oman
}

Received 18 April 2011; accepted 24 September 2011

$$
\begin{aligned}
& \text { فيزيائية تفكك أعمدة و شرائح السوائل }
\end{aligned}
$$

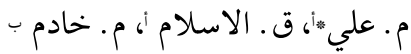

$$
\begin{aligned}
& \text { الخلاصة: يتم البحث باستخدام طريقة عددية في تفاصيل عمليات تفكك أعمدة وشرائح السوائل من أجل التوصل إلى فيزيائية عدم استقرار }
\end{aligned}
$$

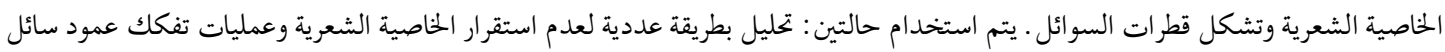

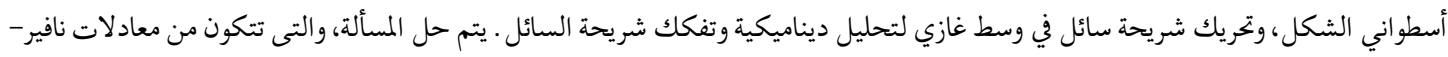

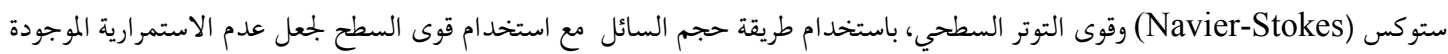

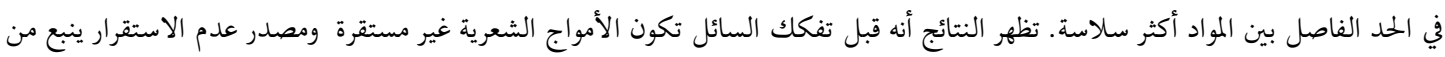

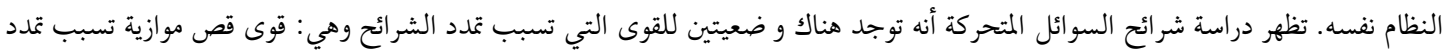

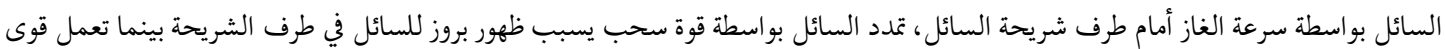



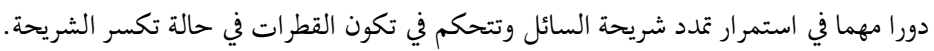

$$
\begin{aligned}
& \text { المفردات المفتاحية : الأمواج الشعرية، تفكك، شرائح السوائل، تكون القطرات، طريقة حجم السائل }
\end{aligned}
$$

\begin{abstract}
The details on breakup processes of liquid column and sheet are numerically investigated to provide the physics of the capillary instabilities and formation of liquid drops. Two cases are used: A numerical analysis on the capillary instabilities and breakup processes of a cylindrical liquid column and a moving liquid sheet in a moving gaseous medium to analyze the dynamics and breakup of the liquid sheet. The problem, composed of the Navier-Stokes systems associated with surface tension forces, is solved by the Volume of Fluid (VOF) technique with a Continuum Surface Force (CSF) to artificially smooth the discontinuity present at the interface. The results show that before disintegration of the liquid the capillary waves become unstable and the source of making the wave unstable is inherently developed by the system. The investigation of moving liquid sheet showed that the two modes of forces for liquid stretching exists: shear force causing the stretching of liquid by shear velocity and drag force causing the stretching of liquid by gas velocity ahead of the tip of the liquid sheets. Stretching of liquid by shear force causes the protrusion of liquid from the tip of liquid sheet and the surface tension force causes the tip of the sheet to make it round. It can also be revealed that the aerodynamic force at the tip of the sheet plays an important role to continue the stretching of sheet and controls the formation of droplet with the occurrence of sheet breakup.
\end{abstract}

Keywords: Capillary wave, Disintegration, Liquid sheet, Liquid atomization, VOF method

\section{Introduction}

Liquid fuel atomization is one of the key for technological challenges in efficient mixing of fuel with oxidizer in both low and high speed transports.

*Corresponding author's e-mail:mali@me.buet.ac.bd
Efficiency of combustion systems and reduction of pollutants are dependent strongly on the degree to which fuel and oxidizer are mixed before they react. No doubt, the mixing of liquid fuel depends strongly on the subsequent fuel atomization technique and the 
characterization of the relevant processes for its distribution in the oxidizer. Since the process of atomization is very quick and fine, a numerical simulation is performed to understand the relevant physics as well as the mechanism of atomization. Moreover the problem is of considerable fundamental interest in the fluid mechanics for a time dependent free-boundary problem and in flow-induced deformation of a variety of flexible bodies. Several methods were proposed and in use for the simulation of such flow problems. These methods are discussed in several published literatures. Gueyffier et al. (1999) described a numerical scheme for interface calculations. The authors used the volume of fluid interface tracking method and a piecewise linear interface calculation in the scheme. The method of interface tracking with the connection of volume fraction and interface position was described in detail. A model called continuum surface force (CSF) for surface tension effects on fluid motion was developed (Brackbill et al. 1991). The model interpreted the surface tension as a continuous, three-dimensional effect across an interface, rather than as a boundary value condition on the interface. Hirt et al. (1981) reviewed different methods used for embedding free boundaries and compared the relative advantages and disadvantages of these methods. A new technique in the volume of fluid (VOF) method was introduced which worked well for complex problems. Welch et al. (2000) used a VOF based interface tracking method in conjunction with a mass transfer model and used in simulation of horizontal film boiling problem.

In an investigation of axisymmetric free-surface flow, Eggers (1993) investigated the characteristic behaviour of drop formation from a liquid column and found that the breaking of fluid neck was independent of the microscopic structure of the fluid, rather it was hydrodynamic phenomena. To understand the dynamics in the immediate viscinity of the point of liquid breakup, Eggers (1995) performed another study on theory of drop formation by analyzing the motion of liquid column using Navier-Stokes equations. An asymptotic solution was developed for both before and after singularity of breaking point. After the singularity, the fluid consisted of two halves, which constituted a unique continuation of the Navier-Stokes equation through the singular point. The nature of the singularities was also investigated in detail by (Eggers and Dupont 2004) using a one-dimensional equation of motion derived from the Navier-Stokes equations for the velocity and the radius during the formation of drop. In an experimental investigation, Kowalewski (1996) studied the details of the shape of the thin liquid neck joining the droplet to its parent body in terms of the fluid viscosity and its jet diameter. The author showed that initially the tip of the jet close to the droplet became convergent, elongated and formed a structure called macro-thread. The tip of the jet began to become thin and created the second, thin microthread between the macro-thread and the droplet. It was found that the maximum length of micro-thread was strongly dependent on liquid viscosity and the retraction velocity after the breakup exhibited relatively small variation with viscosity. An elaborated review work on the drop formation and breakup dynamics was performed by (Eggers 1997) and outlined some unresolved problems on it. Both theoretical development as well as the experimental investigations in this field was reviewed. The work was motivated by the expectation that the pinching was universal under quite general circumstances, even if the motion farther away from the singularity was more complicated. In the non-asymptotic regime, the author focused on the axisymmetric case of a jet with or without gravity which excluded nonlinear free-surface motion, such as drop oscillations, the dynamics of fluid sheets, and the field of surface waves. For swirling jet the variation of thickness of liquid sheet with radius and the dependency of Weber number for disintegration and energy loss in the formation of disintegration were discussed. Similar observation on the behavior of thin liquid sheet were made by (Lin and Roberts 1981; Mehring et al. 1997; Mehring and Sirignano 1998), the later two by using a simplified one-dimensional theory based on the assumption of thin sheets. Lin and Roberts (1981) performed an experiment on the wave motion created by a small obstacle placed on a viscous liquid curtain. Excellent agreements were found between the theoretical study performed by Lin (1981) and experimental results. A considerable difference between the dynamic surface tension and the usual static surface tension was found for a liquid solution.

Recently, Ali (2010) performed an investigation on the dynamics of liquid sheet using VOF method with CSF model. All the above researchers used VOF method for two-phase flow problems with the conjunction of some other models and techniques. Therefore, it can be concluded that the VOF method is one of the popular schemes for tracking interfaces and hence implemented in present algorithm to reveal the physics of dynamics and disintegration processes of liquid column and sheet.

\section{Mathematical Modelling}

The flow field is governed by time dependent threedimensional Navier-Stokes equations with surface tension force. Body forces are neglected. These equations can be expressed as

$$
\frac{\partial \vec{U}}{\partial t}+\frac{\partial \vec{P}}{\partial x}+\frac{\partial \vec{Q}}{\partial y}+\frac{\partial \vec{R}}{\partial z}=\frac{\partial \vec{P}_{v}}{\partial x}+\frac{\partial \vec{Q}_{v}}{\partial y}+\frac{\partial \vec{R}_{v}}{\partial z}+\vec{F}_{s v}
$$




$$
\begin{gathered}
U=\left(\begin{array}{l}
\rho \\
\rho u \\
\rho v \\
\rho w
\end{array}\right), P=\left(\begin{array}{l}
\rho u \\
\rho u^{2}+p \\
\rho u v \\
\rho u w
\end{array}\right), Q=\left(\begin{array}{l}
\rho v \\
\rho u v \\
\rho v^{2}+p \\
\rho v w
\end{array}\right), R=\left(\begin{array}{l}
\rho w \\
\rho u w \\
\rho v w \\
\rho w^{2}+p
\end{array}\right) \\
P_{v}=\left(\begin{array}{l}
0 \\
\tau_{x x} \\
\tau_{x y} \\
\tau_{z x}
\end{array}\right), Q_{v}=\left(\begin{array}{l}
0 \\
\tau_{x y} \\
\tau_{y y} \\
\tau_{y z}
\end{array}\right), R_{v}=\left(\begin{array}{l}
0 \\
\tau_{z x} \\
\tau_{y z} \\
\tau_{z z}
\end{array}\right), F_{s v}=\left(\begin{array}{l}
0 \\
\sigma \kappa f n_{x} \\
\sigma \kappa f n_{y} \\
\sigma \kappa f n_{z}
\end{array}\right)
\end{gathered}
$$

Where, $u, v, \mathrm{w}$ are velocities in the flow field, $\rho$ is the density, $p$ is the pressure, $\pi$ is the viscosity, $F_{s v}$ is the surface tension force, $\sigma$ is the surface tension, $\kappa$ is the curvature of surface, $n$ is the unit normal to the surface and $f$ is a function for continuous change of density across the thickness of fluid interface.

To understand the phenomena of the capillary instability and disintegration processes of liquid column and sheet, a numerical algorithm has been developed to solve time dependent three-dimensional NavierStokes equations with surface tension force. The algorithm can capture the capillary waves radiated on the surface of cylindrical liquid column. For the simulation, VOF method with a fixed, regular, uniform grid is used to solve the problem. Piecewise Linear Interface Calculation (PLIC) is implemented for the advection of liquid interface. The treatment of surface tension consisted of artificially smoothing the discontinuity present at the interface is using Continuum Surface Force (CSF) method Brackbill et al. (1991).

\section{Results and Discussions}

The present unsteady three-dimensional numerical scheme is used to simulate the contraction of a square cylindrical liquid column in an otherwise quiescent gas as well as the capillary waves formed on the liquid gas interfaces. The schematic of the unperturbed square cylindrical liquid column is shown in Fig. 1. The width of the cylinder, " $b$ " is $2.2 \times 10^{-4} \mathrm{~m}$ and the column length and width ratio, $L / b$ is 17.32 . For convenience and easy understanding the average radius of the cylinder is calculated as the square root of (cross sectional area $/ \pi$ ) and denoted as " $a$ ". The average diameter " $d$ " is then calculated as $d=2 \mathrm{a}$. Therefore, the ratio of length and average radius, $L / a$ is 30.73 . Sulfur hexafluoride $\left(\mathrm{SF}_{6}\right)$ having critical temperature of $318.7 \mathrm{~K}$ and critical pressure of $3.76 \mathrm{MPa}$ is used as liquid. Gaseous nitrogen is used as immiscible, viscous fluid with pressure of 7.0 MPa.

\subsection{Capillary Wave and Drop Formation}

The time evolution of capillary wave and droplet

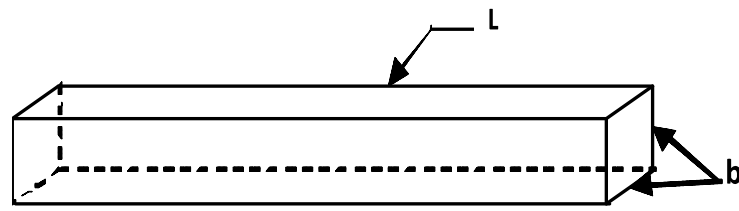

Figure 1. The unperturbed square cylindrical liquid column

formation during the contraction of liquid cylinder is shown in Fig. 2. The dimensionless time " $t$ " has been calculated by the ratio of real time and the timescale, $t_{c}=\sqrt{ } \rho \mathrm{l} r^{3} \backslash \sigma$, where, $\rho l$ is the density of liquid, $r$ is the average radius of the liquid cylinder and is the surface tension. Initially the tip of the liquid cylinder is rectangular in shape. The surface tension force at the sharp edge of the liquid cylinder tip initiates the formation of capillary wave. It can be pointed out that a capillary wave travels along the interface between two fluids of different densities. The dynamics of the capillary waves are dominated by the effects of surface tension. Due to surface tension the cubic end of liquid cylinder changes gradually and forms capillary wave between the interface of liquid and gas with a bulbous shape (like an electric bulb) at the end of liquid column.

From the beginning of calculation, the formation of capillary wave starts and a complete wave including swell and neck forms at about dimensionless time, $t=$ 2.8. The wave then propagates and generates second wave at dimensionless time, $t=5.4$. The propagation time for second wave is shorter than that of first wave. The time interval for the generation of third, fourth, fifth and sixth wave is shorter than that of their predecessors. The dimensionless time of these wave generations are equal to $7.9,10.3,12.4$ and 14.2 , respectively. This uneven time interval for wave propagation is caused by the surface tension force initiated at the end of liquid cylinder. According to the characteristics of the capillary wave, the propagating wave has shorter wavelength than that of the previous wave. Naturally the amplitude of the first wave (wave at the tip of the liquid column) is always greater than that of the following waves. However, the amplitude of the first wave gradually becomes larger and forms a droplet at time, $t=18.2$.

\subsection{Disintegration of Liquid Sheet}

The schematic of the moving liquid sheet with initial setting and calculation domain is shown in Fig. 3. The liquid used for moving sheet is same as liquid column. The moving, quiescent and viscous gas medium is nitrogen with a pressure of 7.0 MPa. The thickness of the liquid sheet is $0.8 \mathrm{~mm}$. The initial length of the liquid sheet, considered from the tip of the sheet to the base, is four times of the half of the unperturbed sheet 

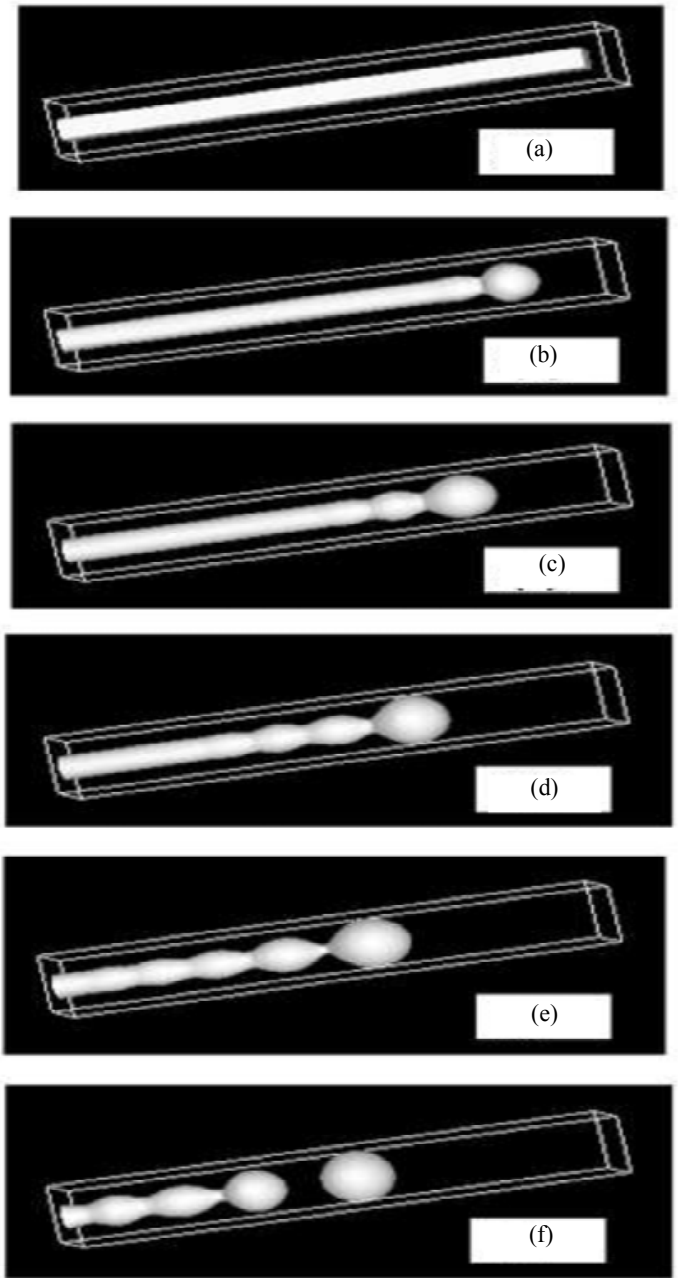

Figure 2. The evolution of capillary wave and liquid disintegration; (a) At dimensionless time, (a) $t=0.0$, (b) $t=4.2$, (c) $t=8.4$, (d) $t=12.6$, (e) $t=16.8$, and (f) $t=21.0$

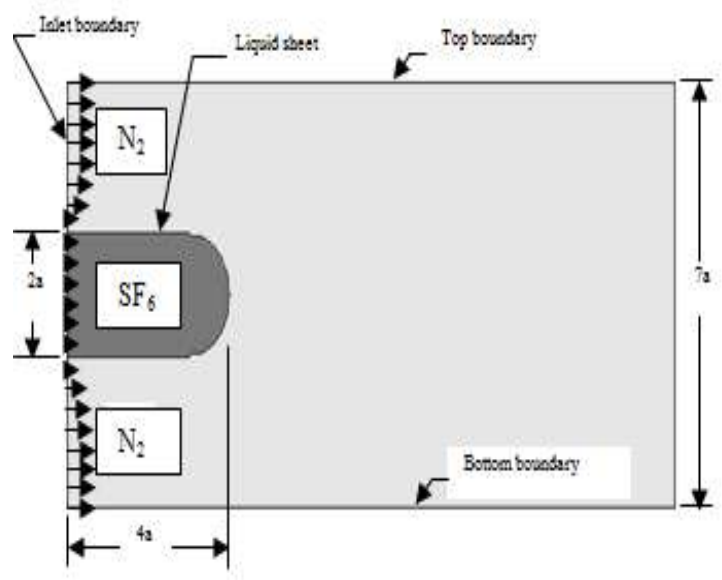

Figure 3. Schematic of unperturbed liquid sheet; a)= half of liquid sheet thickness

thickness " $a$ ". To remove the computational error, the tip of the liquid sheet is initially considered as round. In present study, both the liquid and gas are entering into the flow field from left boundary of the calculation domain. The inlet velocity of liquid and the starting velocity in the liquid in the domain are determined from liquid Weber number $\left(W e_{l}\right)$. The velocity distribution of liquid sheet at the inlet is rectangular as shown in Fig. 3 and uniform velocity distribution is set for liquid region before starting of calculation. Similarly the gas velocity for the inlet boundary and that for the gas in domain is determined from gas Weber number $\left(W e_{g}\right)$. Parabolic velocity distribution at the inlet is considered as shown in Fig. 3 and uniform velocity distribution is set for gas region at the beginning of calculation. For parabolic velocity distribution of gas, the boundary layer thickness is calculated by using the distance of 10 times longer than the liquid sheet thickness. In this study the liquid Weber number, $W e_{l}=1.0$, is considered and for all through the calculation it is kept constant. During simulation, the uniform gas velocity as well as constant gas pressure is imposed on top and bottom boundaries of the calculation domain which is same as inlet gas velocity and pressure. For liquid inlet boundary the constant liquid pressure is used. The zero gradients of both velocity and pressure are used for outlet boundary of the calculation domain.

Figures 4-6 show the arbitrarily magnified velocity vector field with the horizontal velocity on the midsection of the liquid sheet at different dimensionless time. It can be pointed out that the horizontal velocity on the mid-section of the flow field is named as the horizontal velocity along $x$-axis for convenient presentation of the result. Both color and length of arrow in Figs. 5 and 6 indicate the magnitude of the velocity vector at different locations of the flow field. The red color has the highest magnitude of the velocity and the blue has the lowest. However, in different velocity vector field the length of arrow sign shows different magnitude of the velocity. The dimensionless velocity along $x$-axis is superimposed in each figure. It can be pointed out that the horizontal axis is made dimensionless by the ratio of actual distance to the half thickness of the unperturbed liquid sheet and the velocity is made dimensionless by the ratio of actual velocity to the square root of $\sigma /(\rho 1 a)$, where $\rho 1$ is the density of the liquid. The liquid velocity along the $x$-axis of the sheet as well as the gas velocity at the tip of the sheet can be understood from the curve.

Irrespective to the presence of gas flow, it is a common characteristic of liquid that the tip of liquid sheet tends to contract by surface tension. However, the large shear stress acting from the gas side on both sides of the liquid sheet tends to make the liquid sheet thinner. Two effects are activated on the flow field, namely: aerodynamic effect and surface tension effect. Figure 4 shows that due to existence of shear velocity 
at the interface of liquid and gas, the surface of the liquid sheet is stretched. Due to shear velocity, the surface liquid has higher velocity than inner liquid velocity, and changes the direction of flow at the end of the sheet. The streams of surface liquid, coming from either surface of the liquid sheet, merge with each other and form a protrusion of liquid by jet action because the resulting stagnation pressure at the tip is so high that the surface tension acting on the round tip of the initial liquid sheet cannot confine the impinging liquid in the initial liquid sheet. At the same time, a pair of large recirculation (at negative central velocity region) develops inside the liquid sheet near the base of the liquid protrusion. Thus, the main function of aerodynamic effect is to make vortices and a stagnation point. An interesting feature is that, once the recirculation flow is established, the volumetric flow rate of the liquid entering the protrusion region is almost fixed. Figure 4 also shows that the highest liquid velocity towards positive $\mathrm{x}$-direction occurs in the liquid protrusion region. This velocity is about four times greater than the velocity of the liquid entering the calculation domain while the protrusion thickness is about one fourth of the initial liquid sheet thickness. This fact implies that the amount of liquid entering the calculation domain flows into the protrusion region. This trend continues in the subsequent time, so that the length of the liquid sheet with the same magnitude of thickness as the initial one does not change significantly throughout Figs. 4-6.

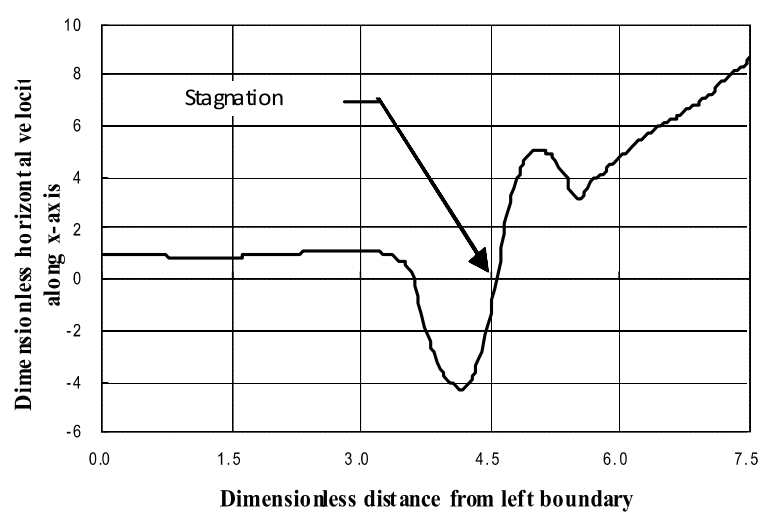

Figure 4. A segment of velocity vector field and hori -zontal velocity along $x$-axis at $t=0.54$ when $W e_{g}=25$

For more details on the formation of the protrusion region, the following fluid dynamic structure can be noted. The increase and decrease in liquid pressure, respectively caused by the surface tension acting on the convex and concave surface part of the liquid sheet produces centrifugal forces necessary for the liquid surface flow to turn along the stepped surface. Therefore, the liquid entering the protrusion region

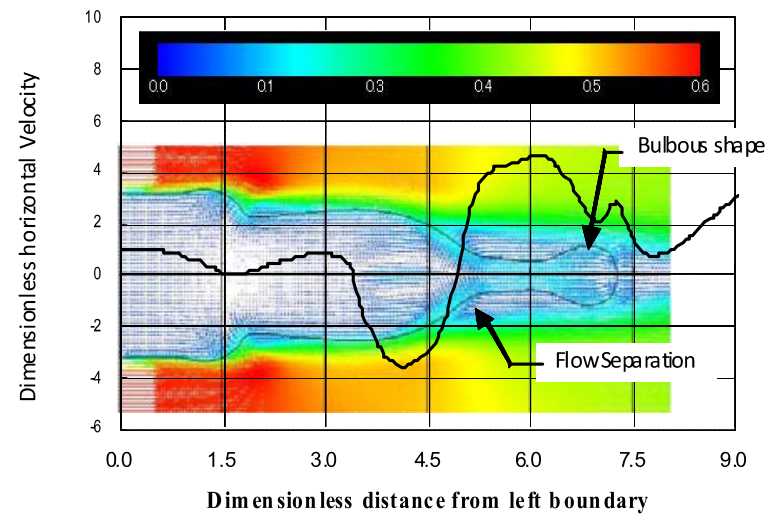

Figure 5. A segment of velocity vector field and horizontal velocity along $x$-axis at $t=1.08$ when $W e_{g}=25$

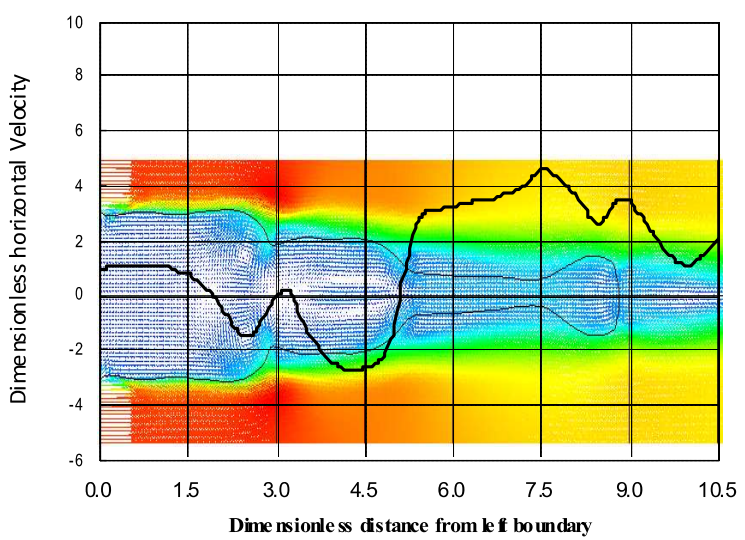

Figure 6. A segment of velocity vector field and horizontal velocity along $x$-axis at $t=1.73$ when $W e_{g}=25$

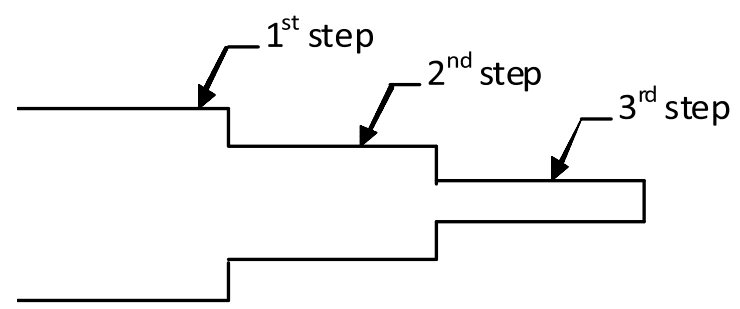

Figure 7. The structure of steps tends to develop by aerodynamic effect

continues to elongate the protrusion region. On the other hand, the surface tension tends to contract the tip of protrusion and forms a surface wave due to which the tip of the protrusion attains a bulbous shape (the tip looks like as an electric bulb) shown in Fig. 5 and gradually increases the diameter of the swell. It is notable that in the protrusion region, the effect of surface tension is intensified because the thinner liquid sheet is embedded in the gaseous wake flow of the much thicker liquid sheet. The velocity in the protrusion region increases towards the tip and then decreases a little at the bulbous tip of the sheet due to a pair of recirculation formed by gas velocity. The horizontal 


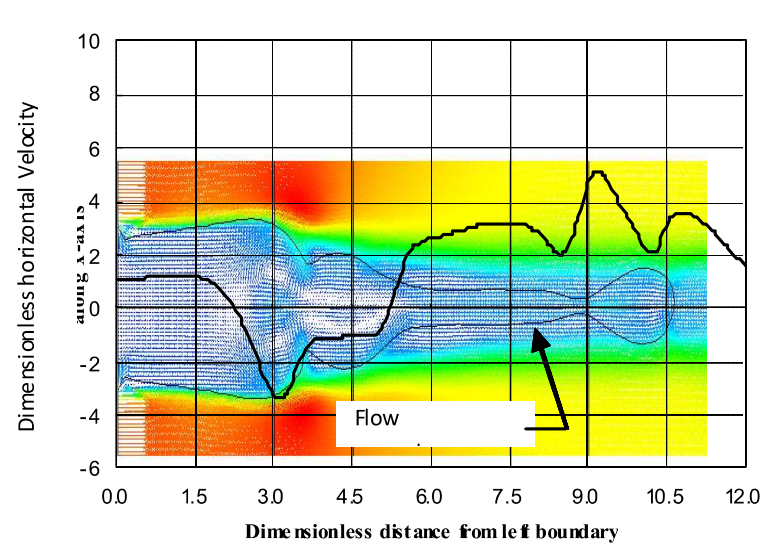

Figure 8. A segment of velocity field and horizontal velocity along $x$-axis at $t=2.37$ when $W e_{g}=25$

velocity distribution also shows that the gas velocity increases continuously with the distance from the tip of the protrusion region along the horizontal direction. The reason can be explained in the way that at initial stage of the flow, the protrusion length is small and the two gas streams coming from opposite sides of the liquid sheet are merging to a common stream at the liquid sheet tip and increase the velocity. This flow sucks the tip and helps stretching the liquid protrusion as shown in Fig. 6.

Figure 5 shows more deformation of the liquid sheet than that of Fig. 4 and the remarkable change of velocity can be observed at the end of first step at location of dimensionless distances from $1.2 \sim 2.5$ and at liquid protrusion region. The figure contains three steps deformation on liquid sheet surface. The first step is close to the liquid sheet entrance, the second step is at the end unperturbed liquid sheet (at dimensionless distance of 4.5), and the third step is the protrusion of liquid which contains a bulbous shape of liquid at the tip caused by surface tension. This step structure looks like stairs as shown in Fig. 7. At the beginning of calculation, a pair of recirculation is produced near the tip of the sheet and makes one step. Due to initial condition of the flow field associated with the inflow conditions of both liquid and gas another step is formed at downstream of the liquid entrance. The formation of step causes the flow separation of gas near the outer edge of each step. Thus, most of the protrusion region is embedded in the separated flow region and experiences the shearing stress directing in the upstream direction.

A weak propagation of capillary wave and contraction of liquid sheet caused by surface tension can be observed in Fig. 8. The development of a neck adjacent to the bulbous tip, at which the first breakup of the liquid sheet will take place at a later time of $t=2.61$, is apparent at this stage. The highest liquid velocity towards positive $x$-direction occurs at the neck, which is the consequence of mass conservation. The first step moves downstream as the new liquid enters the calculation domain. The central velocity distribution in Fig. 6 shows increase of velocity towards negative direction in the first step and decrease of negative velocity in second step. Another observation is that the shape of the liquid sheet tip in Fig. 6 tends to become flat. The change in trend of gas flow at downstream of the protrusion tip can be found in Fig. 5, which is caused by the merging action of two gas streams coming from the opposite side of the protrusion and their separation. With the increase of liquid protrusion length as well as the diameter of the bulbous shape at the tip, the separation length from the tip of sheet increases due to vitiation of main gas streams. This flow separation from the protrusion tip can be observed in Fig. 5 in which little decrease of central gas velocity at distance of 5.5 from left boundary can be found. The flattening of the protrusion tip is caused by the gas flow impinging on the tip. Figure 8 is the velocity vector field at dimensionless time, $t=2.37$ which is near to the breakup time, $t=2.61$. The flow of gas separated at second step, tends to reattach with the liquid surface at the later half of the protrusion part. Due to surface tension effect and the dynamical behaviour of gas to form step, the upstream portion of the neck tends to form a pair of weak recirculation and a stagnation point which cause high pressure and low velocity in the upstream region of neck as shown in Fig. 8. Due to the recirculation and flow stagnation, the neck is destabilized and breaks up.

\section{Conclusions}

A numerical analysis has been performed to investigate the mechanism of both liquid column and sheet breakup and the dynamics involved in the breakup processes. At first, the capillary instability and breakup characteristics are studied during relaxation of a square cylindrical liquid column. Calculation shows that capillary wave generates from the tip of liquid cylinder due to the effect of surface tension force. From the initial stage the swell-growth of the first wave (tip wave) is much higher than that of the following waves which is caused by the surface tension force on the liquid cylinder tip. The propagation of capillary waves towards the base of the liquid column causes a disturbance on the system and makes the wave unstable. Due to instability of the wave, the amplitudes of both the swell and neck grow rapidly and eventually pinch-off occurs.

By the second part of this investigation i.e. breakup mechanisms of liquid sheet, it can easily be understood that the co-flowing gas stretches the liquid sheet and produces the protrusion of the liquid from the tip of the sheet by jet action of the stretching liquid. Due to stretching and protrusion of liquid, steps like stairs 
are formed on the sheet surface. The gas then acts on the steps and forms a pair of vortices and stagnation point near the end of each step. It can be found that the vortices and stagnation point play the main role for the breakup of the liquid sheet. The results show that the capillary wave takes the initiation to form the step by gas flow for breakup. It can be observed that the thickness of liquid protrusion is small and surface tension generates the capillary wave on the tip of the protrusion.

Naturally, a capillary wave contains swell and neck. This neck helps the flow of gas to form a step on the neck. Finally the step is followed by a pair of weak vortices and stagnation pressure which resist the flow of liquid through the thin sheet and eventually the breakup occurs.

\section{Acknowledgments}

The authors gratefully acknowledge the computing facilities in the department of Mechanical Engineering, Bangladesh University of Engineering and Technology (BUET) to conduct this research work.

\section{References}

Ali M (2010), Dynamical behavior of liquid sheet with co-flowing gas. Project Report, CASR, Bangladesh University of Engineering and Technology, April

Brackbill JU, Kothe DB, Zemach C (1991), A continuum method for modeling surface tension. J. Computational Physics 100:335-354.

Eggers J (1993), Universal pinching of 3D axisymmetric free-surface flow. Physical Review Letters 71(21):3458-3460.
Eggers J (1995), Theory of drop formation. Phys. Fluids 7(5):941-953.

Eggers J (1997), Nonlinear dynamics and breakup of free-surface flows. Review of Modern Physics 69(3):865-929.

Eggers J, Dupont TF (1994), Drop formation in a onedimensional approximation of the navier-stokes equation. J. Fluid Mech 262:205-221.

Gueyffier D, Li J, Nadim A, Scardovelli R, Zaleski S (1999), Volume-of-fluid interface tracking with smoothed surface stress methods for three-dimensional flows. J. Computational Physics 152:423456.

Hirt CW, Nichols BD (1981), Volume of fluid (VOF) method for the dynamics of free boundaries. J. Computational Physics 39:201-225.

Kowalewski TA (1996), On the separation of droplets from a liquid jet. Fluid Dynamics Research 17: 121-145.

Lin SP (1981), Stability of a viscous liquid curtain. J. Fluid Mech. 104:111-118.

Lin SP, Roberts G (1981), Waves in a viscous liquid curtain. J. Fluid Mech. 12:443-458.

Mehring C, Kim I, Sirignano WA (1997), Symmetric distortion of a thin liquid sheet with infinite length. Proc. $10^{\text {th }}$ Ann. Conf. Liquid Atom. Spray Sys, 17-121, ILASS, North and South America.

Mehring C, Sirignano WA (1998), Nonlinear capillary wave distortion and disintegration of thin planar liquid sheets. Proc. 11 th Ann. Conf. Liquid Atom. Spray Sys. 155-159, ILASS, North and South America.

Welch SWJ, Wilson J (2000), A volume of fluid based method for fluid flows with phase change. J. Computational Physics 160:662-682. 